\title{
A new approach to the assessment of anaerobic metabolism: measurement of lactate in saliva
}

Metabolism and

Nutrition Unit,

Department of

Physiological Sciences

and Nutrition, Medical

School, University of

Barcelona, Barcelona,

Spain

$R$ Segura, professor

C Javierre,

research associate

M A Lizarraga,

research assistant

E Garrido,

research assistant

Medical and

University Campus of

Bellvitge, University of

Barcelona,

L'Hospitalet de

Llobregat (Barcelona),

Spain

J L L Ventura,

medical staff

Department of Public

Health, Medical

School, University of

Barcelona, Barcelona,

Spain

B Campos,

assistant professor

Correspondence to:

R Segura, Metabolism and

Nutrition Unit, Department

of Physiological Sciences and

Nutrition, Medical School,

University of Barcelona, A.

Diagonal 643, 08028

Barcelona, Spain.

Accepted for publication

13 May 1995

\author{
R Segura, C Javierre, J L L Ventura, M A Lizarraga, B Campos, E Garrido
}

\begin{abstract}
Objective-To test the hypothesis that saliva lactate concentrations may reflect those present in blood and that saliva lactate can be used as a very convenient and useful variable in the study of anaerobic metabolism.

Methods-Parallel determinations were made of lactate in saliva and in capillary blood samples, obtained at 3 min intervals from nine individuals during the performance of a maximum graded exercise test on a cycle ergometer against increasing workloads (from 25 up to a maximum of $300 \mathrm{~W}$ ). Lactate determinations were done by means of an electroenzymatic method using $25 \mu \mathrm{l}$ samples in both types of fluids. Results-For each situation, the concentration of lactate in saliva was shown to be about $15 \%$ of that in plasma but it followed the same pattern of evolution during the exercise test. A good correlation $(r=0.81)$ between blood and saliva lactate concentrations was found. The precision of the method was very good, with a coefficient of variation ranging $(n=10)$ between $2.2 \%$ for samples with very low lactate concentrations and $0.7 \%$ for sample with moderate lactate concentrations. Lactate appeared to be very stable in saliva over a period of 40 days after collection, when kept at $4^{\circ} \mathrm{C}$. The values obtained after this period were virtually identical to those shown in fresh samples.

Conclusions-Determination of lactate in saliva can be used as an alternative to determination in blood, overcoming most of the drawbacks of the procedures being used at present, since the collection of the samples required no special expertise.

(Br f Sports Med 1996;30:305-309)
\end{abstract}

Key terms: saliva lactate; blood lactate; anaerobic threshold; repeated sampling

Despite numerous controversies about the physiological meaning of their changes, ${ }^{1-5}$ serial determinations of plasma or blood lactate constitute a very useful procedure in both experimental and routine studies of physical performance.

To determine the concentration of plasma or blood lactate, samples obtained from a peripheral vessel (such as an antecubital vein) or from the finger tip or the ear lobe are commonly used. Because of the close correlation in the lactate content between samples obtained by arterial or venous catheterisation and those obtained from the ear lobe or the finger tip, ${ }^{6-8}$ "pricking" procedures are generally preferred. In any case, however, the collection of the sample requires some technical expertise and involves a more or less invasive, traumatic, manipulation that may cause stress and anxiety and thus discourage the participants and limit the possibilities of adequate serial sampling, apart from the problems associated with blood handling and manipulation.

We have developed a new procedure that uses saliva as a fluid in which lactate concentrations mirror those in blood. This procedure overcomes most of the drawbacks of the methods presently used, does not require special expertise, and even would allow the subjects to obtain their own samples in practically any place or circumstance.

\section{Methods}

SUBJECTS

A group of nine amateur sportsmen, practising an average of five hours of sporting activities per week and with an acceptable level of physical fitness, agreed to participate in the study after the objectives of the investigation and the protocol to be followed were explaining to them. The main characteristics of the participants were as follows: age 22.2 (SD 1.9) years, height $178.5(7.0) \mathrm{cm}$, and body mass 71.9 (5.8) $\mathrm{kg}$.

All participants consumed their customary (normal) diets and performed their habitual professional and recreational activities. The different trials took place in the afternoon, a minimum of three hours after lunch, and were conducted in a well ventilated room with an ambient temperature of $20-24^{\circ} \mathrm{C}$ and a relative humidity ranging between $45 \%$ and $55 \%$.

PROTOCOL

Each subject performed a graded cycle ergometer test on a Monark ergometer, model $818 \mathrm{E}$ (Monark AB, Sweden). The subjects pedalled at 60 revolutions $\mathrm{min}^{-1}$ for consecutive periods of 3 min duration, interrupted by resting periods of $1 \mathrm{~min}$ which was used for the collection of the saliva and capillary blood samples. The first period, consisting of $3 \mathrm{~min}$ of unloaded pedalling, was followed by successive periods of 3 min duration in which step power output increments of $25 \mathrm{~W}$ up to the maximum that could be achieved by each participant (up to $225 \mathrm{~W}$ in one case, up to $250 \mathrm{~W}$ in three cases, up to $275 \mathrm{~W}$ in four cases, and up to $300 \mathrm{~W}$ in two cases). 
Once each workload step was completed, saliva and blood capillary samples were collected. In order to stimulate the production of saliva, before sampling each subject rinsed his mouth with $5 \mathrm{ml}$ of a dilute solution of citric acid $(0.2 \mathrm{M})$ which was expelled $5 \mathrm{~s}$ afterwards. From that time and for the following 30 $s$ the secreted saliva was continuously collected in a glass funnel placed on top of a graduated cylinder. The subject then washed his mouth with drinking water to remove any traces of the chemical stimulant.

The capillary blood samples were obtained from the ear lobe after briefly "milking" the tissue to move more blood into the area and "pricking" the overlaying skin with a mechanical device. The glass capillary tubes were filled with the flowing blood at the same time that the subject was spitting the saliva into the collection probe. The total time involved in the saliva and blood sample collections was less than $1 \mathrm{~min}$.

As well as sampling at the end of each $3 \mathrm{~min}$ step, saliva and capillary blood samples were obtained at 3,6 , and $9 \mathrm{~min}$ of the recovery period.

Along the graded exercise test and during the recovery phase, the heart rate was continu- ously monitored by means of a Polar Sport Tester (Polar Electro OY, Finland).

\section{ANALYSES}

All samples were analysed for lactate content by means of an enzymatic method using lactate oxidase immobilised within a three-layer membrane which fits into a platinum electrode (YSI 23L blood lactate analyser, model 1500 Sport, YSI Inc, USA). The amount of sample used for the lactate determinations was the same for the saliva and for the capillary blood specimens $(25 \mu \mathrm{l})$. In order to reduce the variability to a minimum and avoid any possible interferences, the clear transparent upper layer of the saliva samples was used for the lactate determination. This clear layer was easily obtained after centrifugation of the sample for a few minutes or after letting the sample settle for 15-20 min.

To estimate the precision of the method, duplicate analyses of the same sample were performed in 33 specimens. To estimate the intra-assay variability, three specimens containing increasing amounts of lactate $(0.3 \mathrm{mM}$, $0.7 \mathrm{mM}$, and $1.8 \mathrm{mM}$ ) and corresponding to three different workloads were analysed 10 times each.
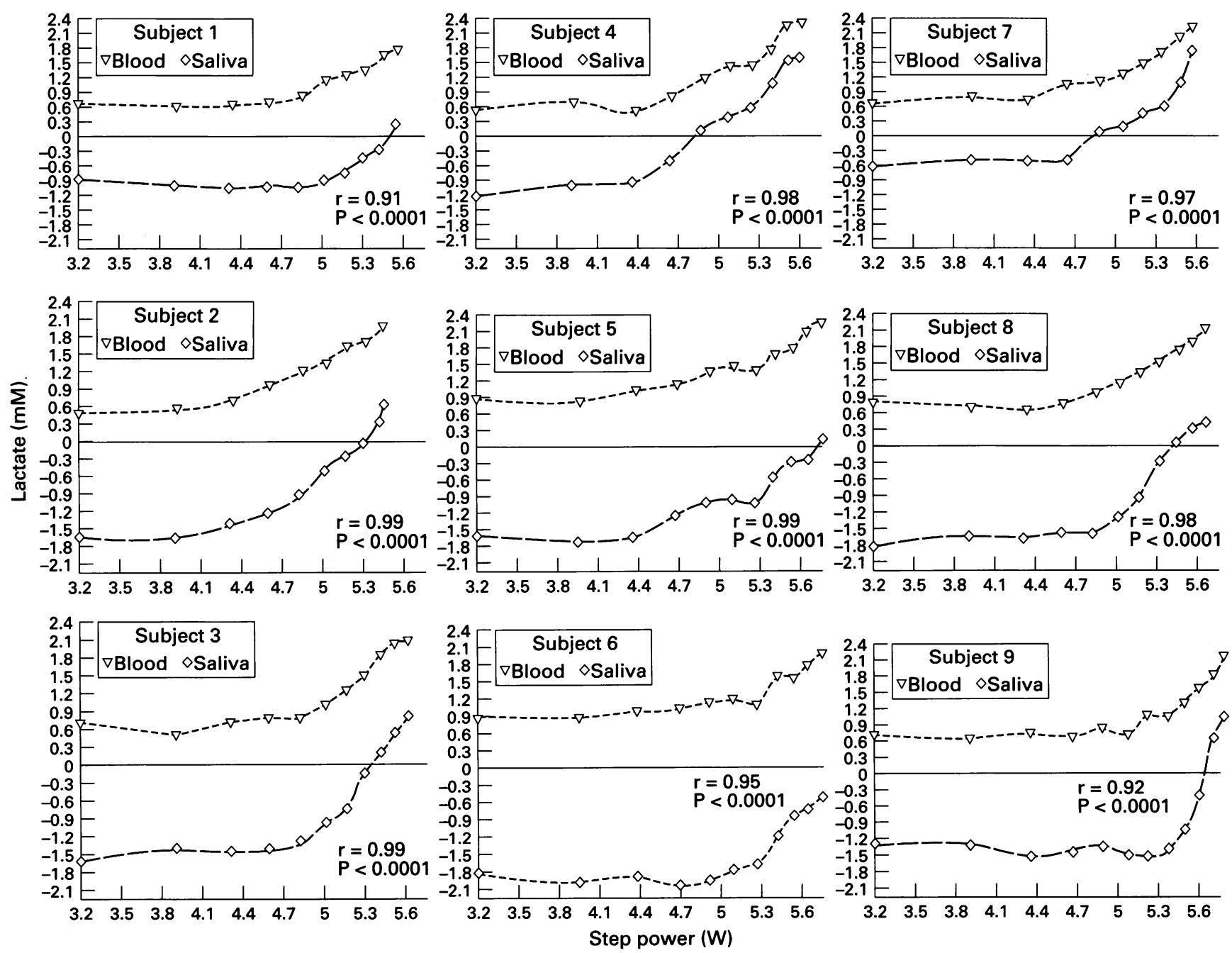

Figure 1 Ln of the concentration of lactate in blood $(\nabla--\nabla)$ and in saliva $(\diamond--\diamond) v$ the ln of the power output (In-ln model) for each of the nine individuals studied. 


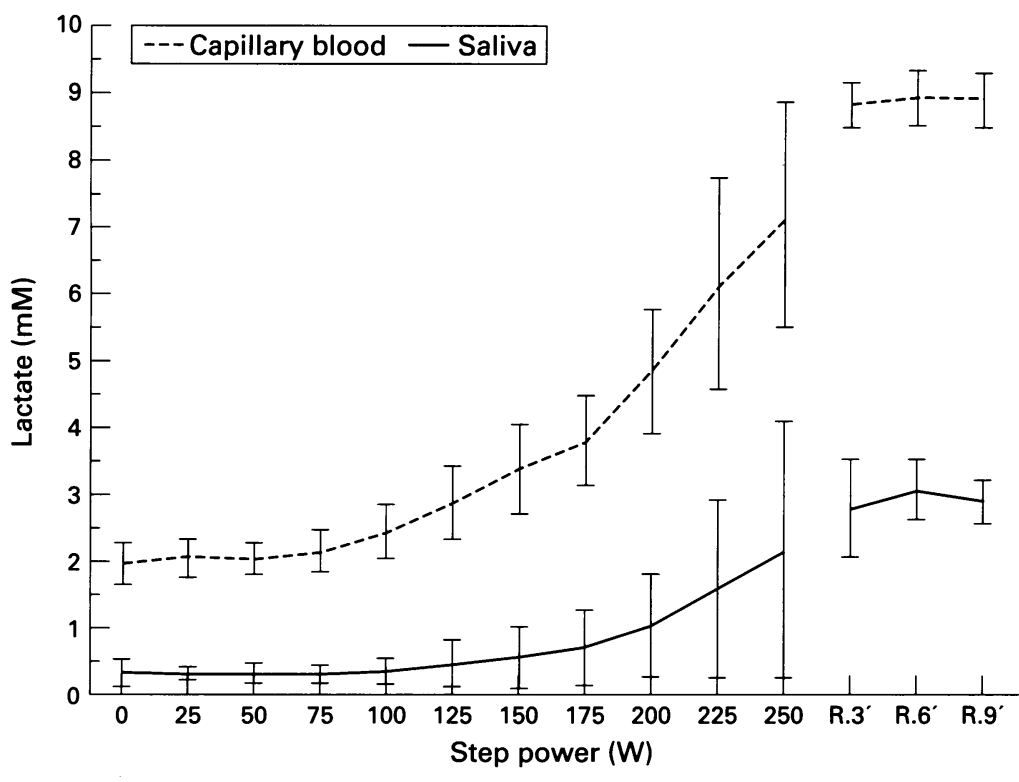

Figure 2 Average lactate concentration in blood and in saliva versus power output in the group of individuals shown in fig 1 . (Mean values and SD for each workload.)

To test the stability of the lactate in the saliva samples, 30 specimens with different concentrations of lactate were reanalysed 40 days after collection, having been kept in the interval in a refrigerator at $4^{\circ} \mathrm{C}$.

\section{STATISTICAL EVALUATION}

We used a two phase linear regression equation $^{9-10}$ to fit the lactate response to increasing workloads according to the log-log model of Beaver et al ${ }^{1-4}$; we used this model because it seems the best available to define the "inflexion" or transition zone between mainly aerobic and anaerobic metabolism.

The equations of the overall regression model, of non-linear character, may be written $^{11}$ as follows:

$E=\left(y_{1}\right)=\theta+\beta_{1}\left(x_{1}-\gamma\right) I=1, \ldots \ldots \ldots ., \tau$

$\mathrm{E}=\left(\mathrm{y}_{1}\right)=\theta+\beta_{2}\left(\mathrm{x}_{1}-\gamma\right) \mathrm{I}=\tau+1, \ldots \ldots \ldots, \mathrm{n}$

where $x_{1}<\ldots \ldots .<x_{\tau}<\gamma<x_{\tau+1}<\ldots . .<x_{n}$ are the ordered logs of the step output power, and $y_{1}$ 's the logs of the lactate concentrations; $\beta_{1}$ and $\beta_{2}$

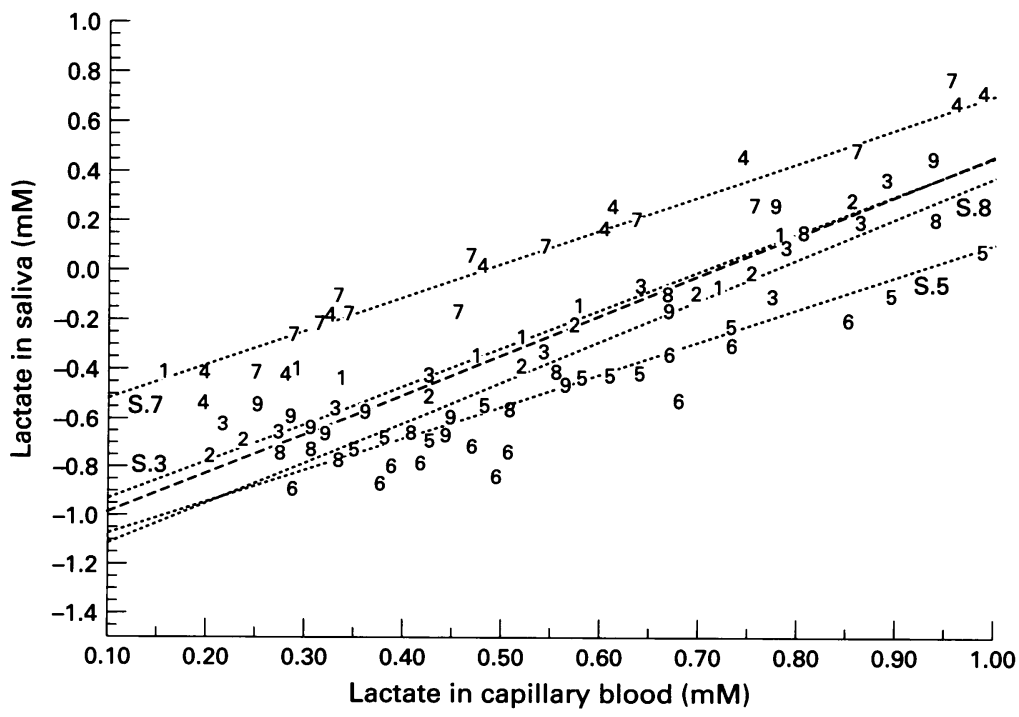

Figure 3 Degree of concordance between the lactate concentration in capillary blood and in saliva samples obtained at the same time for each work load in the nine subjects studied. are the slopes of the two lines; $(\gamma, \theta)$ are the coordinates of the joint point; and $\tau$ is the cutoff point which divides the dataset. These five parameters were estimated following the algorithm detailed by Vieth. ${ }^{12}$

The agreement between the lactate curves obtained from blood and saliva samples in the estimation of the lactate threshold was measured by means of the concordance correlation coefficient. ${ }^{13}$. A $95 \%$ confidence interval for this parameter was estimated to test the ability of the new saliva based method to reflect the threshold values obtained with the classical blood based procedures.

\section{Results}

As can be seen in fig 1, there was a good correlation for each individual, on each workload step, between the concentration of lactate in the saliva samples and in the blood capillary samples. There was also a very good correlation for the whole group of individuals between the mean lactate values in saliva and in capillary blood for each increasing workload (fig 2).

When applying the two phase linear regression equation to the blood and saliva lactate response to increasing workloads, there was a linear relation between the natural logarithm (ln) of the lactate concentration and the $\log$ of the power output with a defined slope, in aerobic conditions. This relation showed a markedly different slope when the metabolic activity moved into progressively anaerobic conditions, the changeover point in the slopes of the two regression lines determining the lactate threshold (LT) or anaerobic threshold (AT). This model was tested against a single straight line model and a significant improvement was found $(P<0.0001)$. The lactate values observed in blood samples and in saliva samples show a high degree of concordance $(r$ $=0.813, \mathrm{P}<0.05,95 \%$ confidence interval 0.49 to 0.96 ) (fig 3 ) The absolute lactate values were lower in the saliva than in the blood samples but they followed a parallel evolution along with the increasing work rate. It is worth noting that the "inflexion" points in the lactate curves constructed from values obtained in the blood are very similar to those obtained from saliva samples, suggesting that this fluid can be used as an alternative to blood sampling in the estimation of the lactate or anaerobic threshold.

The amount of saliva collected during the 30 $s$ intervals between each workload step was quite large, with a mean value of $3.8 \mathrm{ml}$, and a range between 2 and $6 \mathrm{ml}$. Thus, if desired, multiple determinations can be performed on the same sample and-as shown below-after a long period of time has elapsed from the collection day. No problems were encountered during the collection of the samples or afterwards.

Lactate appeared to be very stable in the saliva samples for up to 40 days after collection, the values observed in 30 specimens being almost identical to those obtained in the fresh samples (the variation being mainly due to the instrumental variability). In our hands, 
the precision of the method-tested by repeatedly $(n=10)$ analysing three different saliva samples, corresponding to three different workloads and with increasing concentrations of lactate (mean values : $0.303 \mathrm{mM}, 0.697$ $\mathrm{mM}$, and $1.791 \mathrm{mM}$ ) - was shown to be very good (table). A coefficient of correlation of 0.997 for values obtained by duplicate analyses in 33 saliva specimens was obtained, with no significant differences between repeated determinations analysed by the Student test for paired observations.

\section{Discussion}

Saliva has been used as a diagnostic tool in many clinical situations in which it has provided valuable information about the biochemical, metabolic and functional status of the individual. ${ }^{14}$

Saliva sampling has the advantage over venous or capillary sampling that is less stressful, sparing the uncomfortable and sometimes traumatic experience of repeated venepunctures or skin prickings. It also does not require special training and offers the possibility of the subjects collecting their own samples, saving technicians' time and thus making the whole procedure convenient and economical. These features make repeated sampling more acceptable to the individual, allowing a more detailed and accurate estimation of the biochemical changes related to a particular exercise test, particularly in field studies.

Because of the absence of blood cells in the sample, which could induce changes in the lactate concentration, it is not necessary to separate the liquid from the cellular and other solid constituents of saliva after the collection of the specimen. The lactate values of the individual saliva samples remained practically constant for up to $\mathbf{4 0}$ days after collection.

The concentration of lactate in saliva is several times lower than in plasma, corresponding to approximately $15 \%$ of that observed in blood samples obtained at the same time from the ear lobe. However, the rate of change in the saliva lactate with increasing workloads paralleled that observed in the plasma, though with lower values, in a manner similar to that described for other plasma components such as like cortisol. ${ }^{15}$ There thus appears to be a good correlation between the concentration of lactate in saliva and that in plasma collected at the same time. Moreover the curves constructed from lactate values obtained from saliva and from capillary blood show very similar inflexion points (fig 2), although the slopes of the blood lactate curves are steeper than those corresponding to the lactate in saliva..

We resorted to chemical stimulation of salivary secretion with the aim of achieving the

Precision of the lactate measurement in saliva (repeated determinations $(n=10)$ in three samples)

\begin{tabular}{llll}
\hline & Sample 1 & Sample 2 & Sample 3 \\
\hline Mean (range) & $0.303 \mathrm{mM}$ & $0.696 \mathrm{mM}$ & $1.791 \mathrm{mM}$ \\
& $(0.29-0.31 \mathrm{mM})$ & $(0.68-0.71 \mathrm{mM})$ & $(1.78-1.81 \mathrm{mM})$ \\
SD & 0.0067 & 0.0083 & 0.0119 \\
CV & $2.2 \%$ & $1.2 \%$ & $0.7 \%$ \\
\hline
\end{tabular}

$\mathrm{CV}$, coefficient of variation.
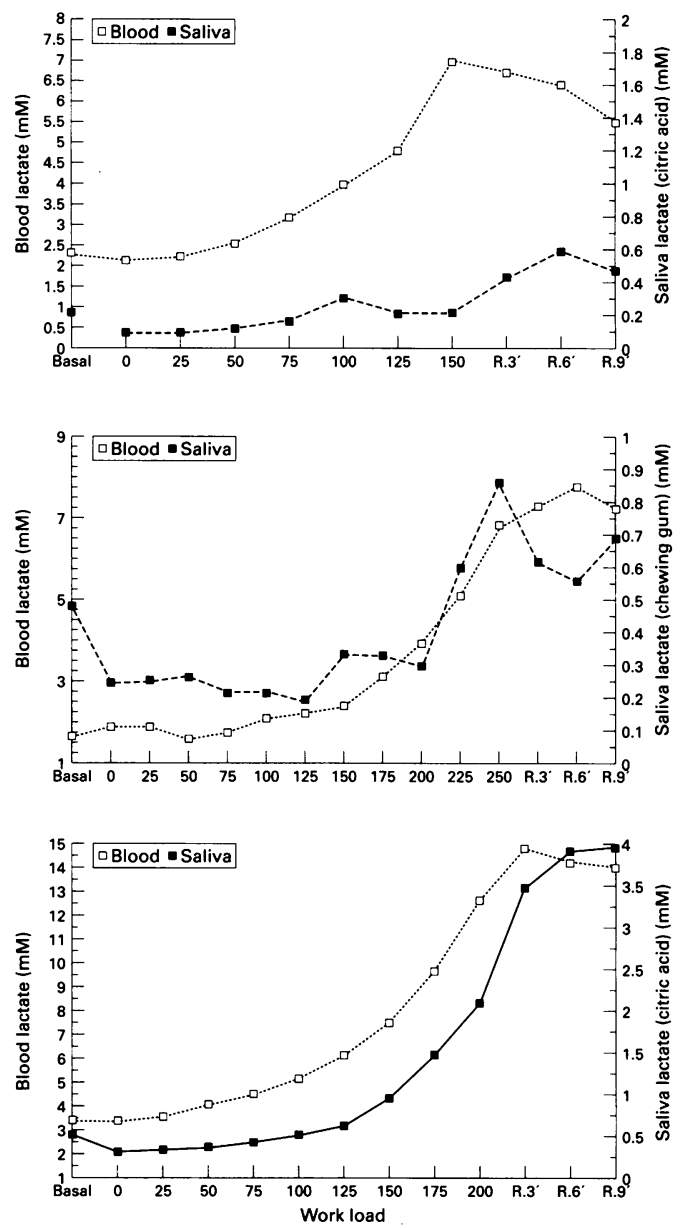

Figure 4 Evolution of lactate concentrations in blood and in saliva samples obtained after mechanical (chewing gum) or chemical (citric acid) stimulation of saliva flow.

highest possible flow rate, as it is known that the composition of saliva is closest to that of plasma when the rate of secretion is increased to a maximum. ${ }^{12}$ At low flow rates, more time is given for the removal of ions and the "remodelling" of the primary fluid, while at higher rates of flow, less time is available for this elaboration, in which case the composition of the saliva is closer to that of plasma.

In order to minimise any possible interferences in the process of transference of lactate from blood to saliva, and with the additional objective of achieving a more homogeneous salivary flow rate, we stimulate the production of saliva using a dilute solution of citric acid which interacts with taste receptors in the tongue and other receptors in the oral mucosa. In the few experiments in which we resorted to mechanical stimulation of saliva secretion (chewing gum) the values obtained were very erratic, with a lesser degree of correlation between the samples obtained in the saliva and those obtained at the same time in capillary blood samples (fig 4). Because the $\mathrm{pH}$ values of stimulated saliva remain within a relatively narrow range, whereas those of unstimulated saliva are more variable, the saliva to plasma lactate ratio should be expected to be more constant after chemical stimulation with citric acid.

The results obtained in our experiments show that the determination of lactate in saliva, 
using the same methods that are used for measuring lactate in blood samples, can be used as an alternative in the assessment of the response of the individual to increasing workloads and in the evaluation of some of the metabolic changes that take place during the performance of physical exercise. However, we cannot be certain that the pattern of saliva lactate response that we have observed following the exercise protocol described in this paper applies to exercises performed following other protocols in which the work rate may change more rapidly.

1 Beaver WL, Wasserman K, Whipp BJ. Improved detection of lactate threshold during exercise using a log-log transformation. ₹ Appl Physiol 1985;59:1936-40.

2 Katz A, Sahlin K. Regulation of lactic acid production during exercise. $\mathcal{F}$ Appl Physiol 1988;65:509-18.

3 Stainsby WN. Biochemical and physiological basis for Stainsby WN. Biochemical and physiological basis
lactate production. Med Sci Sports Exerc 1986;18:341-3.

4 Wasserman K, Beaver WL, Davis JA, Pu J, Heber D, Whipp BJ. Lactate, pyruvate and lactate/pyruvate ratio during exercise and recovery. $\mathcal{f}$ Appl Physiol 1985;59:935-40.
5 Wasserman K, Beaver WL, Whipp BJ. Mechanisms and patterns of blood lactate increase during exercise in man. Med Sci Sports Exerc 1986;18:344-52.

6 Mcloughlin P, Popham P, Linton RAF, Bruce RC, Band D. Use of arterialized venous blood sampling during incremental exercise tests. F Appl Physiol 1992;73:937-40.

7 Oyono-Enguelle S, Gartner M, Marbach J, Heitz A, Ott C, Freund $\mathrm{H}$. Comparison of arterial and venous blood lactate kinetics after short exercise. Int $\mathcal{f}$ Sports Med 1989;10:1624.

8 Williams JR, Armstrong N, Kirby BJ. The influence of site sampling and assay medium upon the measurement and interpretation of blood lactate responses to exercise. $\mathcal{f}$ Sports Sci 1992;10:95-107.

9 Hudson DJ. Fitting segmented curves whose join points have to be estimated. $\mathcal{A}$ Am Stat Assoc 1966;61:1097-129.

10 Sprent P. Some hypothesis concerning two-phase regression Sprent P. Some hypothesis concerning
lines. Biometrics 1967;17:634-45.

11 Hinkley DV. Inference in two-phase regression. $7 \mathrm{Am}$ Stat Assoc 1971; 64:736-9.

12 Vieth E. Fitting piecewise linear regression functions to biological responses. F Appl Physiol 1989;67:390-6.

$13 \mathrm{Lin} \mathrm{LIK}$. A concordance correlation coefficient to evaluate reproducibility. Biometrics 1989;45:255-68.

14 Mandel ID. The diagnostic uses of saliva. 7 Oral Pathol Med 1990;19:119-25.

15 Umeda T, Hiramatsu R, Iwaoka T, Shimada T, Miura F, Sato $T$. Use of saliva for monitoring unbound free cortisol levels in serum. Clin Chim Acta 1981;110:245-53.

16 Thaysen JH, Thorn NA, Schwartz IL. Excretion of sodium, potasium, chloride and carbon dioxide in human parotid saliva. Am $\mathcal{f}$ Physiol 1954;178:155-9. 